\title{
The Additional Value of Three-Dimensional Transesophageal Echocardiography in the Diagnosis of Unusual Complication of Bioprosthetic Mitral Valve
}

\author{
Martina Evangelista, Marta Barletta, Anca Irina Corciu' ${ }^{1}$, Valnetina Mantovani', Lucrezia Delli Paoli', Marco Guazzi ${ }^{2,3}$, Maurizio Tusa ${ }^{1}$ \\ Departments of Cardiology and ${ }^{3}$ Biomedical Sciences for Health, I.R.C.C.S. Policlinico San Donato, University of Milan, Departments of ${ }^{1}$ Cardiology and ${ }^{2}$ Cardiology \\ University, I.R.C.C.S. Policlinico San Donato, San Donato Milanese, Milan, Italy
}

\section{Abstract}

Primary tissue failure of bioprosthetic mitral valves due to cusp perforations or ruptures is an unusual complication on short-term follow-up. An 88-year-old male with a known history of mitral regurgitation (MR) treated with bioprosthetic valve replacement in 2016 was referred to our center for recurrent heart failure. The two-dimensional (2D) transthoracic echocardiography documented an intraprosthetic jet of regurgitation without identifying a clear morphological mechanism, nor quantifying precisely the mitral insufficiency. 3D transesophageal echocardiography (TOE) with the tool FlexiSlice added relevant information by providing insights into the pathophysiological mechanisms of MR. The present case emphasizes the importance of 3D TOE as a fundamental tool for the diagnostic algorithm of bioprosthetic valves failure, even in the more demanding cases.

Keywords: Bioprosthetic valve, flail, mitral regurgitation, three-dimensional transesophageal echocardiography

\section{INTRODUCTION}

Bioprosthetic heart valves have limited durability, and hemodynamic deterioration due to primary tissue failure can occur. Primary tissue failure usually refers to calcification of the leaflets of the bioprosthesis, with or without leaflet tear. Multiple studies have analyzed the pathological and histological features of primary tissue failure of postmortem or at reoperation explanted bioprosthetic valves identifying leaflet calcifications, stiffening, or disruption as the most common causes of bioprosthetic mitral valve failure ${ }^{[1-3]}$ The risk factors for these degenerative changes are younger age, mitral implant position, and renal failure. ${ }^{[4]}$ No clear differences have been identified in short- or long-term follow-up between bovine pericardial and porcine bioprosthetic valves, ${ }^{[5]}$ even if a slight protective effect has been found in the bovine pericardial valve. ${ }^{[6]}$ Currently, only a few data about cusp perforations, ruptures, or detachment are reported in the literature; $;^{[7-9]}$ none of them relating to a short-term follow-up.

The anatomical aspects of the bioprosthetic valve with its thin leaflets and the presence of prosthetic materials causing

\begin{tabular}{|l|l|}
\hline \multicolumn{2}{|c|}{ Access this article online } \\
\hline Quick Response Code: & Website: \\
\hline & www.jcecho.org \\
\cline { 2 - 2 } & \\
\hline
\end{tabular}

numerous artifacts, such as acoustic shadowing, may lead to difficulties in identification, diagnosis, and quantification of mitral regurgitation (MR) ${ }^{[10]}$ For all the above-mentioned reasons, a multimodality multistep approach is the key for the correct diagnosis with an accurate identification of the morphological mechanism and the precise quantification of valve insufficiency. ${ }^{[10]}$

\section{Case Report}

An 88-year-old male was referred to our hospital for recurrent acute heart failure. He underwent coronary artery bypass graft in 2004, and for a myocardial infarction in 2016, he underwent mitral valve replacement with a 29-mm Carpentier-Edwards bovine pericardial bioprosthesis for severe MR. Postoperative clinical course was uneventful; then, he was rehospitalized for

Address for correspondence: Dr. Martina Evangelista, Via Giacomo Leopardi 39/4, Arese (MI) 20020, Italy. E-mail: martina.evangelista@outlook.com

This is an open access journal, and articles are distributed under the terms of the Creative Commons Attribution-NonCommercial-ShareAlike 4.0 License, which allows others to remix, tweak, and build upon the work non-commercially, as long as appropriate credit is given and the new creations are licensed under the identical terms.

For reprints contact: reprints@medknow.com

How to cite this article: Evangelista M, Barletta M, Corciu AI, Mantovani V, Paoli LD, Guazzi M, et al. The additional value of three-dimensional transesophageal echocardiography in the diagnosis of unusual complication of bioprosthetic mitral valve. J Cardiovasc Echography 2019;29:78-81. 
acute heart failure during recurrence of rapid atrial fibrillation and during recrudescent of chronic obstructive pulmonary disease.

In May 2018, he was rehospitalized for recurrence of acute heart failure and was diagnosed as MR. An echocardiographic multistep approach was applied: first, a complete two-dimensional (2D) transthoracic echocardiography was performed, followed by complete 2D and 3D transesophageal echocardiography (TOE). All the images were digitally recorded with GE Vivid E95, and the FlexiSlice tool has been used when necessary. The images were acquired by the same operator.

The transthoracic echocardiogram documented normal left ventricle volumes and wall thickness, with preserved systolic function (ejection fraction 55\%) and no regional wall motion abnormalities. The aortic valve was tricuspid and continent, with no significant anterograde gradient. The right ventricle had normal wall thickness and preserved longitudinal systolic function Tricuspid anular plane systolic excursion (TAPSE $18 \mathrm{~mm}$ ), with moderate tricuspid regurgitation.

Notably, transthoracic echocardiography identified an apparently severe MR considering the depth and the width of the regurgitant jet, above all taking into account the evident Coanda effect, and the increased E-wave $(1.55 \mathrm{~m} / \mathrm{s})$. The precise diagnosis and an accurate MR quantification have been limited by the apparently small vena contracta $(3 \mathrm{~mm})$ and the impossibility to perform an adequate, effective regurgitant orifice area (EROA), due to the excessively eccentric jet [Figure 1]. Due to the poor acoustic window and the presence of numerous artifacts, no clear information about valve morphology and the pathological mechanism of MR might be obtained.

Conversely, 3D TOE allowed to identify the pathophysiological mechanisms of the MR and FlexiSlice allowed a precise quantification of MR. In this context, the 3D TOE with FlexiSlice tool permitted to assess the severity of the MR by looking at the valve from a parallel plane perfectly aligned to the origin of the regurgitant jet and subsequently measure directly the EROA (obtaining a dimension of $0.6 \mathrm{~cm}^{2}$ ) [Figure 2].

Moreover, the underlying anatomical mechanism of the MR was also unclear at the 2D TOE, and only the $3 \mathrm{D}$ has allowed us to understand the morphological aspects. In fact, once acquired, the 3D dataset can be rotated from both atrial and the ventricular perspectives leading, in this particular case, to identify the coaptation defect in correspondence of one bioprosthetic cusp.

In Figure 3, the liable cusp presents a rupture on its tip and develops a reversal movement in the left atrium during the systole, behaving like a flail with the eccentric regurgitation jet directed toward the lateral atrial wall.

No evidence of mitral stenosis was present; the leaflets were characterized by a normal systolic movement with no evidence

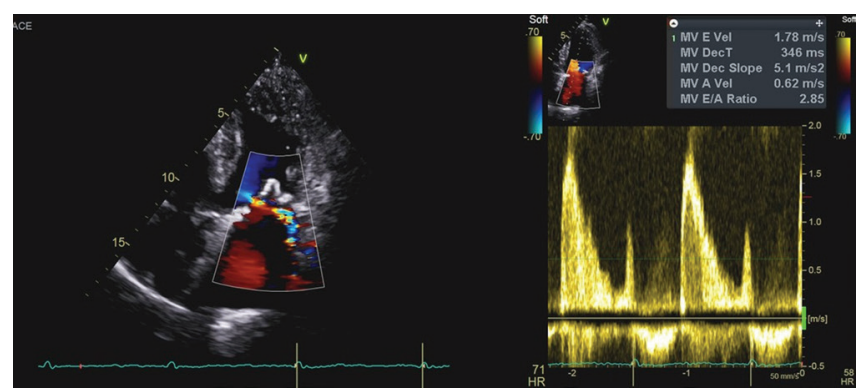

Figure 1: Color Doppler in four-chamber view at transthoracic echocardiography permitted to identify a severe regurgitant jet; the increased E-wave supported the hypothesis that the mitral regurgitation was severe. Due to the excessively oriented jet was not possible to perform the PISA measures

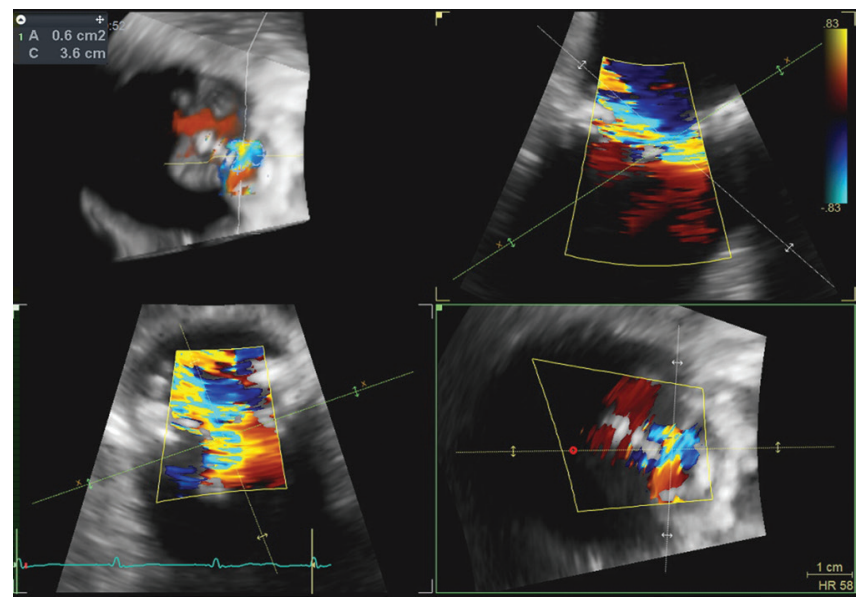

Figure 2: The three-dimensional tool FlexiSlice allows to measure directly the effective regurgitant orifice area

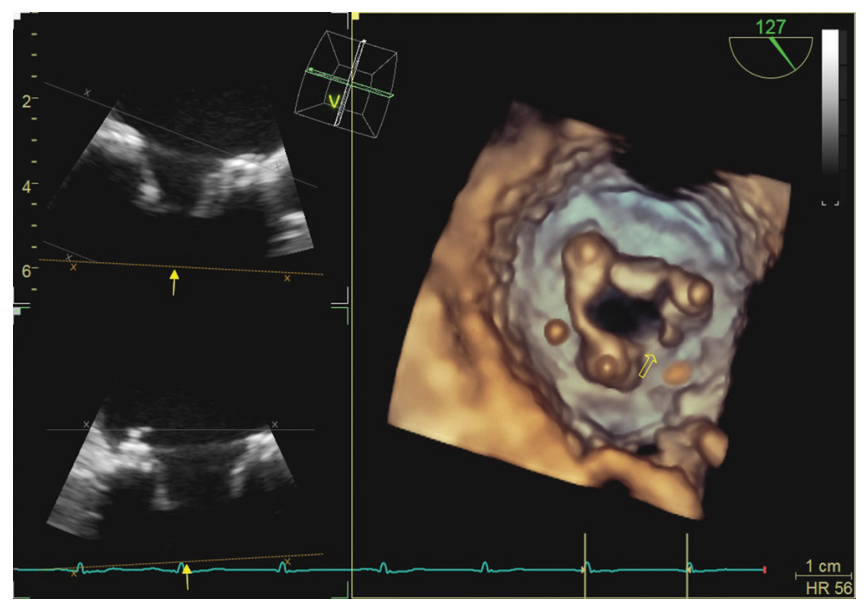

Figure 3: The three-dimensional tool FlexiSlice permits to look at the valve from both the atrium and the ventricle sides, leading to the identification of a coaptation defect of only one cusp

of calcifications nor stiffening (mean pressure gradient: $4 \mathrm{mmHg}$ and peak velocity: $1.76 \mathrm{~m} / \mathrm{s}$ ).

Prostatic cancer, chronic renal impairment, chronic obstructive pulmonary disease, and previous transcatheter ablation of atrial 
Evangelista, et al.: 3D transesophageal echocardiography for the assesment of bioprosthetic mitral valve dysfunction

fibrillation were part of the patient's history. Currently, the patient had a preserved functional status and was physically active. The case was discussed by the heart team who opted for valve replacement. Considering all the comorbidities and the growing evidence of feasibility and safeness of valve-in-valve treatment for bioprosthetic mitral valve degeneration, ${ }^{[11,12]}$ the patient was consensually proposed as a candidate for valve-in-valve treatment of MR.

\section{DISCUSSION}

Primary tissue failure of bioprosthetic heart valves is a possible complication of surgical valve replacement during long- and short-term follow-up with a cumulative incidence of frequency variable from $10 \%-15 \%$ at 10 years to $20 \%-22 \%$ at 20 years. ${ }^{[1-5]} \mathrm{A}$ scheduled echocardiography follow-up is of fundamental importance, especially to detect the rare cases of early complications and failure. Usually, primary tissue failure presents with calcification or stiffening of the leaflets. ${ }^{[1-5]}$ Commissural tear and leaflet perforation associated or not with cusp calcification may be an infrequent presentation. ${ }^{[7,8]}$ Because of its low rate of occurrence, a prompt diagnosis and correct quantification of commissural tear or leaflet perforation are challenging, and a multistep approach is of fundamental importance. During the past decades, echocardiography has become the primary noninvasive imaging method for the evaluation of valvular regurgitation by a combining information on the extent of regurgitant orifice, assessment of the valve anatomy and function, as well as the consequences on cardiac chambers. ${ }^{[13]}$ The aim of a $2 \mathrm{D}$ transthoracic echocardiography is to evaluate prosthetic cusp motion, the presence of calcification and/or abnormal structures (e.g., vegetations, thrombosis) on the various components of the prosthesis, and the integrity of the sewing ring. The Doppler echocardiography may help in the assessment of regurgitation degree using adjunctive parameters such as peak velocity, mean pressure gradient, and estimating the EROA with the use of Doppler signal velocity time integral. Atrial and ventricle chamber dimension and function, as well as estimation of pulmonary artery pressure, may give adjunctive information about the severity of valve dysfunction. ${ }^{[10,13]}$

This set of information, even if plentiful and in some cases redundant, may become insufficient in complex cases of prosthetic valves malfunctioning. For such reasons, TOE and 3D echocardiography have been improved and always more often used in the most challenging diagnosis, ${ }^{[14]}$ showing a great accuracy in the identification of bioprosthetic mitral valve dysfunction. ${ }^{[15]}$ In this case, 2D transthoracic echocardiography has been useless due to the challenging morphological aspects of the valve, the artifacts caused by the prosthetic material and to the poor acoustic windows. 2D TOE has added some information on the valve morphology leading to a hypothetical diagnosis of cusp flail, but the certainty of diagnosis and the precise assessment of valve regurgitation severity needed the 3D TOE with the FlexiSlice tool to be done. In conclusion, in this case, 3D TOE has been of fundamental importance to correctly detect the uncommon pathophysiological mechanism of MR in a bioprosthetic valve and to precisely quantify the severity of the regurgitation.

\section{Declaration of patient consent}

The authors certify that they have obtained all appropriate patient consent forms. In the form the patient(s) has/have given his/her/their consent for his/her/their images and other clinical information to be reported in the journal. The patients understand that their names and initials will not be published and due efforts will be made to conceal their identity, but anonymity cannot be guaranteed.

\section{Financial support and sponsorship}

Nil.

\section{Conflicts of interest}

There are no conflicts of interest.

\section{RefERENCES}

1. Eric Jamieson WR, Marchand MA, Pelletier CL, Norton R, Pellerin M, Anderson WN Jr., et al. Structural valve deterioration in mitral replacement surgery: comparison of carpentier-edwards supra-annular porcine and perimount pericardial bioprostheses. J Thorac Cardiovasc Surg 1999;118:297-304.

2. Bortolotti U, Milano A, Mazzucco A, Valfré C, Talenti E, Guerra F, et al. Results of reoperation for primary tissue failure of porcine bioprostheses. J Thorac Cardiovasc Surg 1985;90:564-9.

3. Marbarger JP Jr., Clark RE. The clinical life history of explanted prosthetic heart valves. Ann Thorac Surg 1982;34:22-33.

4. Fann JI, Miller DC, Moore KA, Mitchell RS, Oyer PE, Stinson EB, et al. Twenty-year clinical experience with porcine bioprostheses. Ann Thorac Surg 1996;62:1301-11.

5. Ganapathi AM, Englum BR, Keenan JE, Schechter MA, Wang H, Smith PK, et al. Long-term survival after bovine pericardial versus porcine stented bioprosthetic aortic valve replacement: Does valve choice matter? Ann Thorac Surg 2015;100:550-9.

6. Grunkemeier GL, Furnary AP, Wu Y, Wang L, Starr A. Durability of pericardial versus porcine bioprosthetic heart valves. J Thorac Cardiovasc Surg 2012;144:1381-6.

7. Aoyagi S, Tanaka K, Nishi Y, Hara H, Kosuga K, Oishi K, et al. Bioprosthetic valvular dysfunction in mitral position: Three cases of commissural detachment. Rinsho Kyobu Geka 1989;9:195-8.

8. Ishihara T, Ferrans VJ, Boyce SW, Jones M, Roberts WC. Structure and classification of cuspal tears and perforations in porcine bioprosthetic cardiac valves implanted in patients. Am J Cardiol 1981;48:665-78.

9. Misawa Y, Oki SI, Tezuka Y, Fuse K. An unusual complication associated with the carpentier-edwards porcine bioprosthesis. ASAIO J 2001;47:563-4.

10. Lancellotti P, Pibarot P, Chambers J, Edvardsen T, Delgado V, Dulgheru R, et al. Recommendations for the imaging assessment of prosthetic heart valves: A report from the European Association of Cardiovascular Imaging Endorsed by the Chinese Society of Echocardiography, the Inter-American Society of Echocardiography, and the Brazilian Department of Cardiovascular Imaging. Eur Heart J Cardiovasc Imaging 2016;17:589-90.

11. Ghosh-Dastidar M, Narayana A, Boix R, Bapat V. Mitral valve-in-valve and valve-in-ring for failing surgical bioprosthetic valves and rings. J Cardiovasc Surg (Torino) 2016;57:372-80.

12. Paradis JM, Del Trigo M, Puri R, Rodés-Cabau J. Transcatheter valve-in-valve and valve-in-ring for treating aortic and mitral surgical prosthetic dysfunction. J Am Coll Cardiol 2015;66:2019-37.

13. Lancellotti P, Tribouilloy C, Hagendorff A, Popescu BA, Edvardsen T, Pierard LA, et al. Recommendations for the echocardiographic 
Evangelista, et al.: 3D transesophageal echocardiography for the assesment of bioprosthetic mitral valve dysfunction

assessment of native valvular regurgitation: An executive summary from the European Association of Cardiovascular Imaging. Eur Heart J Cardiovasc Imaging 2013;14:611-44.

14. Lang RM, Adams DH. 3D echocardiographic quantification in functional mitral regurgitation. JACC Cardiovasc Imaging 2012;5:346-7.
15. Sugeng L, Shernan SK, Weinert L, Shook D, Raman J, Jeevanandam $\mathrm{V}$, et al. Real-time three-dimensional transesophageal echocardiography in valve disease: Comparison with surgical findings and evaluation of prosthetic valves. J Am Soc Echocardiogr 2008;21:1347-54 